\title{
La Formación y Desarrollo Conceptual en el Cálculo Diferencial y el Álgebra Lineal en las Carreras de Ingeniería
}

\author{
Olga Lidia Pérez González \\ olguitapg@gmail.com \\ https://orcid.org/0000-0003-4475-814X \\ Universidad de Camagüey Ignacio Agramonte Loynaz (UCIAL) \\ Camagüey, Cuba
}

Recibido: 28/04/2020 Aceptado: 21/05/2020

\begin{abstract}
Resumen
En el ámbito científico actual de la Comunidad Iberoamericana de Matemática Educativa se desarrolla una tendencia hacia el decrecimiento de trabajos teóricos conceptuales sobre las didácticas particulares de las diferentes asignaturas de Matemática. Desarrollando una investigación cualitativa, apoyada en el método dialéctico materialista, la modelación teórica y el enfoque sistémico estructural, el artículo integra en términos descriptivos los resultados de un proyecto de investigación que tuvo como núcleo teórico la formación y desarrollo conceptual en el contexto del Cálculo Diferencial y el Álgebra Lineal en las carreras de ingeniería. A partir del desarrollo exitoso de cuatro tesis de doctorado, que utilizaron modelos como construcciones teóricas, se caracterizaron los rasgos esenciales de las relaciones, formación y desarrollo conceptual-procedimental en el contexto antes descrito. La investigación obtuvo el premio Nacional de la Academia de Ciencias de Cuba a los resultados de la investigación científica del año 2018 y sus resultados constituyen una propuesta alternativa a las diversas estrategias investigativas de los matemáticos educativos, así como una nueva forma de reflexionar con relación a la Didáctica de esas asignaturas.
\end{abstract}

Palabras clave: Cálculo Diferencial. Álgebra Lineal. Ingeniería. Desarrollo conceptual.

\section{Formação e desenvolvimento conceitual em Cálculo Diferencial e Álgebra Linear}

\section{Resumo}

No atual campo científico da Comunidade Ibero-americana de Matemática Educacional, destaca-se o uma tendência à diminuição de trabalhos teóricos conceituais sobre os didáticos particulares de diferentes disciplinas de matemática. Desenvolvendo uma pesquisa qualitativa, apoiada no método dialético materialista, modelagem teórica e abordagem sistêmica estrutural, o artigo integra em termos descritivos os resultados de um projeto de pesquisa cujo núcleo teórico foi a formação e o desenvolvimento conceitual no contexto do Cálculo Diferencial e Álgebra Linear em carreiras de engenharia. A partir do desenvolvimento bem-sucedido de 4 teses de doutorado que utilizaram os modelos como construções teóricas, foram caracterizadas as características essenciais do treinamento conceitual, desenvolvimento conceitual, desenvolvimento conceitual-processual e o desenvolvimento de relações conceituais no contexto descrito acima. A pesquisa obteve o Prêmio Nacional da Academia Cubana de Ciências pelos resultados da pesquisa científica do ano de 2018 e seus resultados constituem uma proposta alternativa às várias estratégias de pesquisa de matemáticos educacionais, bem como uma nova maneira de refletir sobre a didática desses sujeitos.

Palavras-chave: Cálculo Diferencial. Álgebra Linear. Engenharia. Desenvolvimento conceitual. 


\title{
The formation and conceptual development in Differential Calculus and Linear Álgebra
}

\begin{abstract}
Mathematics education in the Ibero-American Community. However, there is a tendency to the decreasing of conceptual theoretical works on the particular didactics of different Mathematics subjects. This article integrates the results of a research project whose theoretical core was the formation and conceptual development in the context of Differential Calculus and Linear Algebra in engineering careers. The research is descriptive and qualitative, supported by the materialistic dialectical method, theoretical modeling, and the structural systemic approach. Four doctoral theses were used to characterize the conceptualization. All these theses used the models as theoretical constructions and served to characterize the essential features such as the relations, formation and conceptual-procedural development. The research obtained the National Prize of Cuba's Academy of Sciences in 2018 for the results of the scientific research. Its results constitute an alternative proposal to the various research strategies of educational mathematicians, as well as a new way to reflect the Didactic of these subject matters.
\end{abstract}

Keywords: Differential Calculus. Linear Algebra. Engineering. Conceptual development.

\section{Introducción}

En el marco del proyecto "Perfeccionamiento de la enseñanza de la Matemática" del Programa Nacional Problemas Actuales del Sistema Educativo Cubano, perspectivas de desarrollo, coordinado por la autora del presente artículo, y con la participación de un grupo de trabajo multidisciplinario que incluyó profesores de Matemática de las carreras de ingeniería y de preuniversitario, psicólogos, informáticos, ingenieros con categoría docente, y estudiantes de maestría y de doctorado, de las Repúblicas de Cuba y de Dominicana, se realizó la investigación sobre la Didáctica del Cálculo Diferencial y el Álgebra Lineal en las carreras de ingeniería.

Los resultados de la investigación se clasificaron en aportes teóricos-conceptuales y aportes prácticos, y se orientaron a caracterizar los rasgos esenciales de la formación y desarrollo conceptual en esas asignaturas, debido a las insuficiencias detectadas en el tratamiento didáctico de los conceptos y su repercusión en el desempeño de los estudiantes para resolver problemas matemáticos.

En ella se integran coherentemente los resultados de las investigaciones doctorales de Mola (2013), Báez (2018), Báez Ureña (2018) y Martín (2018), así como, las propuestas de Martín, Pérez, Blanco y Casas (2014), Martín, Pérez, Casas, Espíndola y Vargas (2015), Báez, Blanco y Pérez (2015), Báez, Blanco y Pérez (2015b), Báez, Pérez y Triana (2017), Báez, Martínez-López, Pérez y Pérez (2017), Báez, Heredia y Pérez (2017), Farit, Estrada y Ramos (2017), Martín, Pérez, Casa y Sánchez (2017), Nardín, Montalván, Salgado y Pérez (2017), 
Báez, Pérez y Blanco (2018), Bueno y Pérez (2018), Pérez (2018), Pérez y Blanco (2019) y la de Martín y Pérez (2019).

El objetivo del artículo es comunicar en términos descriptivos los aportes teóricosconceptuales de la investigación; a través de la metodología seguida para su desarrollo, con el uso del método dialéctico materialista, la modelación teórica y el enfoque sistémico estructural, y la articulación de los enfoques socioepistemológico y ontosemiótico del conocimiento matemático, las propuestas teóricas sobre los registros de representación semiótica de conceptos matemáticos y los postulados sobre el desarrollo humano desde la perspectiva histórico cultural.

\section{Antecedentes}

El siglo XXI se caracteriza por transformaciones en profundidad en el ámbito de la educación superior; todo ello dado por los cambios que se operan en el ámbito económico, científico, social, cultural y político, lo que ha creado gran expectativa y un movimiento renovador entre los profesores de las asignaturas del área de las ciencias básicas, que ha instado a la comunidad académica del nivel superior a lograr una visión estratégica para el proceso de formación de ingenieros, con el objetivo de prever el panorama en el cual se desempeñarán en el futuro (Vargas, Burguet, Lezcano y Durán, 2018).

En ese sentido, Farit, Estrada y Ramos (2017), King (2012), y Martín y Pérez (2019) plantean que la formación de los ingenieros del futuro deben tener presente los cambios tecnológicos; a su vez, debe enfocarse en el desarrollo de habilidades analíticas de ingenio práctico, a la creatividad, la comunicación, el negocio, la gestión, las normas éticas, el profesionalismo, el dinamismo, la agilidad y la flexibilidad, lo que exige una sólida formación científico-tecnológica, con notable predominio del componente formativo sobre el informativo, y una fuerte formación en ciencias básicas e informática para así aprender a aprender de por vida.

Esa necesidad de generar cambios sustanciales en la formación de ingenieros ha resultado en el desarrollo de algunas investigaciones como la de Rodríguez (2017) y la de Plaza y Villa-Ochoa (2019) quienes han reflexionado sobre los desafíos y retos de la Matemática para estos profesionales en formación, y sobre los obstáculos matemáticos detectados en su formación, todo lo cual sucede en un entorno caracterizado por estrategias de alta tecnología de 
la llamada industria 4.0, referidas al uso de tecnologías digitales en la automatización y digitalización de procesos y negocios, y en la electrónica, entre otros, con su valor agregado orientado a la gestión de la información. Ese contexto de industria 4.0 se caracteriza, como:

Toda la infraestructura física que utilizan sensores y softwares que funcionan en red, para predecir, gestionar, modelar y simular riesgos e impactos en la toma de decisiones en la industria, los negocios y en las organizaciones, sustentado en el desarrollo de sistemas, internet de las cosas, la impresión 3D, la realidad virtual, el Big Data, así como la Inteligencia Artificial, entre otras novedades tecnológicas (Minnaard y Comoglio, 2019, p. 2).

Lo que conduce a repensar en nuevos perfiles profesionales para la formación de los futuros ingenieros para que sean competitivos para la innovación tecnológica.

En las investigaciones llevadas a cabo por Plaza (2016), Rendón-Mesa, Duarte y VillaOchoa (2016), Plaza y Villa-Ochoa (2019), Cordero, Del Valle y Morales (2019) y Mendoza y Escalona (2019) se confirma que para lograr la competitividad en la innovación tecnológica, los ingenieros requieren ser, además, competentes en el trabajo en equipo para la construcción, análisis y/o aplicación de modelos matemáticos, que describan idealmente los distintos aspectos del comportamiento de esas tecnologías, capaces de aplicar los conceptos fundamentales de la matemática para poder deducir las conclusiones cuantitativas y cualitativas de los procesos y fenómenos técnicos, y obtener soluciones simbólicas, gráficas y numéricas de los problemas objeto de estudio sobre la base de los datos experimentales logrando la simulación de estos mediante modelos computacionales.

En ese sentido es que Pérez y Blanco (2019) argumentan la necesidad de formar a los ingenieros para que participen en la solución de problemas reales y trabajen en equipos integrados por especialistas de otras profesiones para construir modelos matemáticos, elegir los métodos matemáticos más adecuados para la solución de problemas y/o analizar modelos matemáticos y sus cálculos aproximados, con auxilio de la tecnología, lo que requiere de un alto rigor en la formación y desarrollo conceptual en la Matemática como vía esencial para su participación independiente y creadora en la resolución de problemas ingenieriles.

Asimismo, los autores anteriormente referenciados hacen énfasis en que el rigor en la formación y desarrollo conceptual en la Matemática es esencial en la formación del ingeniero, y sugieren el análisis de la orientación didáctica de esta asignatura en función de las relaciones, formación y desarrollo conceptual-procedimental contextualizado a la ingeniería. 
Ese rigor ha de lograrse en cada una de asignaturas de Matemática que forman parte del currículo y se orientan al Cálculo Diferencial e Integral de funciones de una y varias variables, el análisis vectorial, el Álgebra Lineal, la Geometría Analítica, Métodos operacionales de cálculo, Probabilidades, Estadística y Variable Compleja, entre otras, que están distribuidas en los primeros semestres o módulos de la malla curricular.

Báez, Martínez-López, Pérez y Pérez (2017) y Martín y Pérez (2019) precisan que el Cálculo Diferencial y el Álgebra Lineal se constituyen en las asignaturas con mayores dificultades didácticas para lograr el rigor requerido en la formación y desarrollo conceptual, por ser las primeras materias que recibe el ingeniero en formación y porque están permeadas por la algebrización de los conceptos, con enfoque aritmético, que predomina en la enseñanza precedente, de ese modo, ellas tienen la misión de abordar didácticamente las rupturas del paso del pensamiento algebraico al pensamiento analítico, así como al desarrollo del pensamiento variacional, característico del pensamiento ingenieril.

En ese sentido, el estudio de Pérez y Blanco (2019) devela la limitada existencia de investigaciones teórico-conceptuales que se enfocan a la didáctica de la Matemática para ingenieros, específicamente en el Cálculo Diferencial y el Álgebra Lineal, en correspondencia con las exigencias actuales de educarlos con una sólida formación conceptual, como vía esencial para la conformación del pensamiento matemático que requiere esta profesión.

Por lo que, las construcciones teóricas orientadas al tratamiento didáctico de los conceptos del Cálculo Diferencial y del Álgebra Lineal para ingeniería, siguen siendo un reto para formar ingenieros con potencialidades de comprender y aplicar los conceptos matemáticos, así como poner de manifiesto la dinámica de las interacciones y transformaciones que se dan entre ellos, y caracterizar sus relaciones como un sistema, lo que conduce a un mejor desempeño en la solución de problemas matemáticos.

A tono con lo anteriormente expresado, investigaciones como las de Nardín, Montalván, Salgado y Pérez (2017), Báez, Martínez-López, Pérez y Pérez (2017), Bueno y Pérez (2018), Martín y Pérez (2019) y otras, prestan atención al perfeccionamiento del tratamiento didáctico de los conceptos Cálculo Diferencial y/o el Álgebra Lineal para ingeniería desde dos perspectivas esenciales, ellas son:

- Una que se orienta a los estudios exploratorios o soluciones prácticas centradas en estrategias, metodologías, acciones, actividades, métodos u otras variantes, 
fundamentadas en diversas teorías didácticas, como la relacionada con la disponibilidad de recursos para la representación y transferencia de registros semióticos (Báez, Pérez y Triana, 2017; Báez, Pérez y Blanco, 2018).

- Y otra que se orienta a los estudios que validan, argumentan y/o desarrollan teorías en proceso de crecimiento, como son los enfoques etnomatemáticos, socioepistemologógicos y ontosemióticos del conocimiento matemático (Arrieta y Díaz, 2015; Camacho-Ríos, 2011).

A su vez, Pérez y Blanco (2019) develan la necesidad del desarrollo de trabajos teóricos conceptuales orientados a investigar las propiedades inherentes y relaciones presentes en las didácticas particulares de las diferentes asignaturas de Matemática, específicamente las del Cálculo Diferencial y el Álgebra Lineal para las carreras de ingeniería, lo que pudiera considerarse como una tercera perspectiva de vital importancia para que no existan desbalances en la relación que debe existir entre los intereses de la universidad, como institución social, y los intereses de la sociedad.

\section{Metodología}

Tomando como referente a León-Duarte (2019) en el artículo se presenta un estudio teórico conceptual que se caracteriza por analizar, comparar, integrar, codificar y relacionar los enfoques socioepistemológico y ontosemiótico del conocimiento matemático, así como las teorías sobre los registros de representación semiótica de conceptos matemáticos, para estudiar la formación y desarrollo conceptual en el contexto del Cálculo Diferencial y el Álgebra Lineal en las carreras de ingeniería. Se utiliza como referente a Godino, Vicenç, Contreras y Wilhelmi (2005) para defender la postura de que la diversidad de teorías es un desafío, y a su vez, es un punto de partida de las investigaciones en el campo de la didáctica.

La concepción dialéctico materialista de la ciencia fue la base para abordar la integralidad de las explicaciones del proceso de formación conceptual en el Cálculo Diferencial y el Álgebra Lineal en el contexto ingenieril, desde la perspectiva de sus orígenes, desarrollo, estructura y movimiento, sus nexos y dinámicas, en movimiento y transformación en las condiciones reales de la práctica educativa y en concatenación con otros procesos o sistemas (Arias, 2018). 
El enfoque sistémico-estructural se utilizó en todas las fases de la investigación y se consideraron como referentes las obras de González (1984) y Marrero y Lasso (2017), para concebir el proceso de formación y desarrollo conceptual en el Cálculo Diferencial y el Álgebra Lineal en el contexto ingenieril, y así estudiarlo como un conjunto organizado e interdependiente de subsistemas en constante interacción, que se van regulando por funciones dinámicas que existen entre ellos, y con el exterior, que se enmarca con otros sistemas que se relacionan en interacción con la sociedad.

Es así que en esta investigación se caracterizan las propiedades cualitativas de su objeto de estudio como formación integral, su comprensión como subsistema de un sistema más amplio, sus partes componentes (subsistemas), estructura interna, funciones y relaciones funcionales que conforman su nueva cualidad.

Por su parte, la modelación teórica se utilizó para sistematizar las teorías precedentes sobre la base de los fundamentos teóricos declarados anteriormente, lo que permitió describir, explicar y argumentar la estructura funcional del objeto de estudio, sus nuevas categorías teóricas y argumentos, sus relaciones o nexos teóricos, a través de su representación gráfica mediante un esquema, las que se convierten en exigencias para modelar la posterior práctica educativa (Valledor, 2019).

La investigación se desarrolló a través de tres fases que se interrelacionaron en determinados momentos debido a la propia lógica de la investigación, ellas son:

- Fase 1: Caracterización del marco teórico para la formación y el desarrollo conceptual en el Cálculo Diferencial y el Álgebra Lineal en el contexto ingenieril

En esta fase se realizó la sistematización teórica sobre la formación y el desarrollo conceptual en la Matemática y se precisaron las principales dificultades del Cálculo Diferencial y el Álgebra Lineal en el contexto ingenieril y la definición de las categorías teóricas de la investigación desde la perspectiva de la formación y desarrollo conceptual.

Para identificar las dificultades se realizaron dos estudios explicativos en las carreras de Ingeniería de la Universidad Autónoma de Santo Domingo en el cuatrimestre septiembrediciembre de 2015 en el Cálculo Diferencial (Báez, 2018) y en Álgebra Lineal (Martín, 2018), como parte de las investigaciones doctorales.

Ambos casos fueron desarrollados en dos momentos, el primero, para caracterizar el desempeño didáctico del docente en la materia objeto de estudio, con énfasis en el tratamiento 
didáctico de la formación y desarrollo de conceptos matemáticos, y el segundo para valorar el desempeño de los estudiantes en la solución problemas matemáticos en el Cálculo Diferencial y el Álgebra Lineal en el contexto ingenieril.

Participaron 125 docentes y 350 estudiantes, seleccionados a través de un muestreo no probabilístico; además de indagar por su disposición positiva para participar en el estudio. Posteriormente se llevó a cabo un análisis documental y les fue aplicado un cuestionario orientado a develar el grado de satisfacción con relación a los métodos y recursos empleados en clases para la formación y desarrollo conceptual y su incidencia en la solución de problemas; y un test para valorar el nivel con que se desempeñan los estudiantes al operar con los conceptos en la solución de problemas matemáticos.

Para valorar la estructura, instrumentos y resultados fueron utilizados diversos métodos estadísticos tales como la prueba de independencia de Chi-cuadrado, el análisis porcentual y las tablas de contingencias posterior a la aplicación del método Delphi y los talleres de socialización (Pérez y Blanco, 2019).

- Fase 2: Construcción de los modelos teóricos

Estuvo orientada a explicar de forma integrada la formación y desarrollo conceptual en el Cálculo Diferencial y el Álgebra Lineal en el contexto ingenieril desde la formación y desarrollo conceptual, el desarrollo conceptual procedimental y el desarrollo de las relaciones conceptuales, a través de subsistemas, sus relaciones e interacciones y sus respectivos componentes, sus funciones y cualidades distintivas.

- Fase 3: Valoración de la pertinencia científica de los modelos teóricos propuestos y sus impactos sociales

El criterio de expertos se utilizó como técnica de consenso, lo que conllevó al procesamiento estadístico de las opiniones recibidas en relación a los resultados teóricos conceptuales de la investigación, en base a la experiencia científica, profesional y al análisis lógico que resulta de la experticia sobre la coherencia y factibilidad de aplicación en la práctica educativa.

La encuesta incluyó la disposición a participar como expertos, la autovaloración del nivel de competencia que tenían en relación a la temática de investigación, aspectos estos 
determinantes para su selección como expertos. La escala para la valoración de los expertos era de muy adecuado, suficientemente adecuado, adecuado, poco adecuado e inadecuado.

Posteriormente fueron concretados los resultados de la modelación teórica en tres estrategias didácticas que se implementaron en la práctica educativa en la Universidad Autónoma de Santo Domingo de República Dominicana.

La valoración de la implementación en la práctica se realizó a través de tres experimentos pedagógicos en ese mismo país, orientados a valorar el desempeño de los estudiantes para resolver problemas matemáticos en el Cálculo Diferencial y el Álgebra Lineal en el contexto ingenieril.

Finalmente, se participó en la convocatoria anual de Premios Nacionales a los resultados de la investigación científica, del año 2018, de la Academia de Ciencias de Cuba, en la Comisión de Ciencias Sociales, como otra vía de valoración de la pertinencia científica de los modelos teóricos propuestos y sus impactos sociales.

\section{Resultados}

\section{Sistematización teórica sobre la formación y el desarrollo conceptual en la Matemática}

Desde los postulados del materialismo dialéctico se define el concepto como una de las formas del reflejo del mundo para penetrar a la esencia de los objetos, fenómenos y procesos; de modo tal que, en la medida que la actividad del ser humano se amplía, su contenido se enriquece, se modifica o se renueva, de ahí su carácter de sistema abierto (Rosental y Ludín, 1981).

De ese modo, él no es una entidad estática, sino que se encuentra en constante desarrollo, orientado a la búsqueda de un mejor reflejo de la realidad a través de la articulación dialéctica del análisis-síntesis, la inducción-deducción y lo empírico-teórico del saber, por lo que su formación y desarrollo se concibe como un proceso complejo que requiere de la aplicación de diversos métodos teóricos como la comparación, la abstracción, la generalización, la flexibilidad y la diferenciación, entre otros.

Es así que Pérez y Blanco (2019) y Ramos (2000) explican la formación y desarrollo conceptual como un proceso de automovimiento en forma de espiral que transita de lo inferior a lo superior con atención a la interacción dialéctica de lo histórico-lógico, y como una actividad 
que se da en la relación sujeto-objeto, en la que interviene lo práctico, lo cognoscitivo, lo valorativo y lo comunicativo.

Báez (2018) precisa que la comprensión de la formación y desarrollo conceptual como actividad requiere de las relaciones dialécticas teoría-práctica y conceptual-procedimental en un contexto histórico-social determinado y bajo la premisa de que el mundo es cognoscible, lo que requiere una concepción didáctica que se oriente a la búsqueda del nexo entre lo conceptual y lo procedimental con flexibilidad, análisis inductivo-deductivo y significatividad.

Los postulados de Vigotsky (1987) son la base para relacionar el proceso de formación y desarrollo conceptual con la experiencia histórico-social del estudiante, y el carácter mediatizado de la psiquis humana a través de la utilización de signos o símbolos, considerados instrumentos mediadores que sirven para externalizar las representaciones mentales, y develar los significados personales relativos a los conceptos objetos de estudio y los significados socialmente compartidos por una comunidad de referencia (en este caso los ingenieros), a través de la interacción social como vía de su materialización y socialización.

Este proceso requiere de la comprensión gradual y progresiva de los conceptos objeto de estudio, atendiendo a las características del medio sociocultural, el tránsito de lo social a lo individual y las vivencias del estudiante orientado, todo lo cual orienta hacia la significatividad de la actividad que realiza el estudiante.

En esta investigación, la "significatividad de la actividad" encuentra su fundamento en la obra de Castellanos, Castellanos, Llivina, Silverio, Reinoso y García (2002), entre otros, quienes precisan que ello sucede cuando se logran relaciones entre los nuevos conocimientos con los conocimientos que ya se poseen (significatividad conceptual), entre la experiencia cotidiana, la del conocimiento y la de la vida (significatividad experiencial) y entre los nuevos contenidos con el mundo afectivo-motivacional del sujeto de la actividad (significatividad afectiva).

En el contexto de la Didáctica de la Matemática se explica que los registros de representación semiótica de los conceptos matemáticos constituyen la materialización de los postulados vigotskianos sobre pensamiento y lenguaje, ya que:

Todo concepto matemático remite a no-objetos; por lo que la conceptualización no es y no se puede basar sobre significados que se apoyen en la realidad concreta; en otras palabras en matemáticas no son posibles reenvíos ostensivos. Todo concepto matemático se ve obligado a servirse de representaciones, dado que no se dispone de objetos para 
exhibir en su lugar, por lo que la conceptualización debe necesariamente pasar a través de registros representativos que, por varios motivos, sobre todo si son de carácter lingüístico, no pueden ser unívocos (D'Amore, 2009, p. 152).

Las representaciones semióticas de los conceptos matemáticos, usando el lenguaje natural, fórmulas algebraicas, gráficos geométricos y otros signos o símbolos, constituyen el medio a través del cual se pueden externalizar las representaciones mentales para hacerla accesible a los demás y valorar su propio pensamiento (Báez, Pérez y Blanco, 2018, p. 20).

Lo anterior requiere de la comprensión de la dualidad proceso-objeto, propuesta por Tall (1991), acerca de que en la Matemática un proceso y un objeto pueden estar representados por un mismo símbolo, lo que requiere de un proceder reflexivo con el uso de las transferencias de registros semióticos de los conceptos matemáticos y de la significatividad del nexo conceptual procedimental.

A partir de los postulados del materialismo dialéctico y del desarrollo sociocultural y cognoscitivo del estudiante, en esta investigación se articulan propuestas teóricas del enfoque socioepistemológico y ontosemiótico del conocimiento matemático, por ser portadoras de la visión sociocultural del desarrollo del conocimiento, que tienen en cuenta el relativismo histórico-cultural de las prácticas y significados matemáticos, considerando esta articulación como un desafío y punto de partida para el desarrollo teórico conceptual que se realiza.

El enfoque socioepistemológico del conocimiento matemático centra su propuesta en la construcción de los saberes matemáticos a partir de prácticas sociales en las que, al decir de Cantoral (2013) y Reyes-Gasperini y Cantoral (2014), se enriquecen los saberes construidos con nuevos significados, de manera que develen los diversos usos del conocimiento matemático a partir del intercambio de las prácticas de referencias y diversidad de contextos para el saber matemático, y hacen énfasis en el desarrollo del pensamiento variacional, con el uso de estrategias y códigos variacionales en la solución de problemas matemáticos.

Al considerar lo anterior, la investigación se centra en el estudio de procesos de variación y cambio a través de la comprensión de sistemas ingenieriles que relacionen variables internas, de manera que se identifiquen patrones de regularidad de dichos fenómenos; así como el desarrollo de acciones cognitivas para reproducir covariaciones entre magnitudes, y la exteriorización de sus representaciones mentales mediante representaciones semióticas que pueden ser en registros geométricos, numéricos, estocásticos y métricos. 
Por lo que, los constructos analíticos del enfoque socioepistemológico sobre las prácticas sociales, la funcionalidad, contextualidad, relativismo y resignificación del conocimiento matemático conducen a la argumentación didáctica del proceso de formación y desarrollo conceptual centrado en prácticas ingenieriles, de forma tal que los conocimientos matemáticos se doten de significados a través de su uso y funcionalidad en los problemas propios de la ingeniería.

El sistema de nociones teóricas y metodológicas que aporta el enfoque ontosemiótico del conocimiento matemático permite argumentar el proceso de formación y desarrollo conceptual en coherencia con los postulados vigotskianos y del materialismo dialéctico, al considerar como centro del proceso a la práctica matemática como acción compartida y situada, intencional y mediada por recursos lingüísticos y materiales.

Desde esa perspectiva Godino, Batanero y Font (2007) argumentan que la Matemática tiene una triple dimensión: una es la práctica matemática de resolución de problemas socialmente compartida, otra es el lenguaje simbólico, y otra es el sistema conceptual lógicamente organizado, para lo que se debe lograr la articulación dialéctica que devele su lógica didáctica en contextos determinados, y con esta la autonomía conceptual en la resolución de dichos problemas.

Lo anterior condujo a Báez Ureña (2018) a considerar, en el Cálculo Diferencial y el Álgebra Lineal en un contexto ingenieril, la articulación dialéctica entre el proceso de adquisición de recursos para la transferencia de registros semióticos en la resolución de problemas matemáticos en el contexto ingenieril, el proceso de formación del lenguaje matemático, y el tránsito del lenguaje coloquial al matemático en la descripción del movimiento de la variable para la formación y desarrollo conceptual..

El sistema de nociones que propone Godino, Vicenç, Contreras y Wilhelmi (2005) relacionado con el sistema de prácticas operativas y discursivas en función de los tipos de problemas (en este caso los ingenieriles) y los significados que a ellas se le atribuyen, los objetos ostensivos y no ostensivos que intervienen en los sistemas de prácticas y los que permiten caracterizarlas (tipos de problemas, procedimientos, lenguaje, procedimientos, definiciones, argumentaciones y proposiciones), y las relaciones y configuraciones entre objetos para relacionarlos entre sí y conformar redes entre ellos, constituyen argumentos para comprender el 
proceso de formación y desarrollo conceptual desde la perspectiva del desarrollo conceptualprocedimental y las relaciones entre conceptos.

Esos mismos autores caracterizan la dialéctica instrumento-objeto en el mismo sentido que lo hace Tall (1991) acerca de la dualidad proceso-objeto explicada anteriormente, pero en los términos de la unidad dialéctica entre el subsistemas de prácticas operatorias (praxis) y discursivas (logos), de manera que favorecen la significatividad del desarrollo conceptual procedimental.

En relación a lo anterior, Martín, Pérez, Casas y Sánchez (2017) argumentan la necesidad de la significatividad de las relaciones conceptuales en la formación del futuro ingeniero, para que se articulen los conceptos desde el nexo logos-praxis, como vía para develar de forma progresiva esas relaciones, su lógica argumentativa y la especificidad significativa del contenido a través de su funcionalidad matemática en la interpretación y análisis de los modelos matemáticos de procesos técnicos y/o en la resolución de los problemas ya modelados.

Al resumir los presupuestos anteriores, se sintetiza que la formación y el desarrollo conceptual en la Matemática requiere comprender la dualidad proceso-actividad caracterizada por la funcionalidad, contextualidad, relativismo y resignificación del conocimiento matemático en la solución de problemas matemáticos, el nexo entre lo conceptual procedimental, el desarrollo de las relaciones conceptuales y la fusión de procesos y conceptos representados por diferentes registros de representación semiótica, donde la flexibilidad, la significatividad y la autonomía conceptual son las propiedades cualitativas que caracterizan su tratamiento didáctico y el desempeño de los estudiantes en la Matemática.

Se requiere, además, del desarrollo del pensamiento variacional en los futuros ingenieros, para que puedan identificar, interpretar y analizar modelos matemáticos de procesos técnicos, económicos, productivos y/o científicos vinculados a la ingeniería haciendo uso eficiente de las tecnologías disponibles para resolver problemas ingenieriles.

\section{Dificultades del Cálculo Diferencial y el Álgebra Lineal en el contexto ingenieril}

Como resultado de los estudios explicativos realizados por Mola (2013), Báez (2018), Báez Ureña (2018) y Martín (2018), en los que participaron 75 docentes y 603 estudiantes, se identificaron las siguientes dificultades en el Cálculo Diferencial y el Álgebra Lineal en las carreras de ingeniería como las causas esenciales del problema de investigación, ellas fueron: 
- Pobre comprensión del movimiento de la variable como parte integral de los objetos y fenómenos del contexto ingenieril y que se manifiesta a través del límite funcional.

- Insuficiente uso de las diversas representaciones semióticas de los conceptos matemáticos y en la transformación de los procesos en objetos.

- Escasa atención al uso y comprensión de las hipótesis bajo las cuales se resuelve cada problema matemático, para lograr correctas aplicaciones conceptuales en el solución de problemas ingenieriles.

- Insuficiente tratamiento didáctico al uso correcto del lenguaje matemático y de la tecnología para la solución de problemas en el contexto ingenieril.

- Predominio de la argumentación geométrica y poca atención al desarrollo del pensamiento variacional para la formación y desarrollo de conceptos matemáticos.

- Poca atención a la identificación de las relaciones conceptuales, y al reconocimiento de los conceptos que la favorecen, para mejorar el desempeño en la solución de problemas matemáticos en el contexto ingenieril y como contexto para la formación y desarrollo de los conceptos matemáticos objeto de estudio.

En síntesis, como resultado de esos estudios explicativos se precisó que la tendencia manifiesta en esas asignaturas se orienta a la sobrevaloración del tratamiento algorítmico con énfasis en la representación y conversión de registros semióticos pero con poca atención a la comprensión de la dualidad proceso-objeto y de los conceptos matemáticos.

\section{Categorías teóricas de la investigación desde la perspectiva de la formación y}

\section{desarrollo conceptual}

Se definieron las categorías teóricas de la investigación: proceso de enseñanzaaprendizaje Cálculo Diferencial y el Álgebra Lineal, formación del concepto, apropiación del concepto, formación conceptual, desarrollo conceptual procedimental, relaciones conceptuales y desarrollo de relaciones conceptuales.

La categoría teórica proceso de enseñanza-aprendizaje del Cálculo Diferencial y el Álgebra Lineal dadas por Báez (2018) y Martín (2018) se definió como aquel proceso innovador que proyecta la aplicación de recursos didácticos que combinan, movilizan y relacionan los 
conceptos y los procedimientos, utilizando la lógica dialéctica entre lo abstracto-concreto, el análisis-síntesis y lo inductivo-deductivo, a través de la representación y transferencia de registros semióticos de los objetos matemáticos, la valoración de las hipótesis, el análisis de los movimientos de la variable, el reconocimiento de patrones variacionales del contexto ingenieril, la argumentación de los procesos de variación y cambio, la representación de las relaciones del objeto algebraico a través de una red identitaria y la consolidación del nexo símbolo-objeto en la resolución de problemas matemáticos aplicados al contexto ingenieril.

Por su parte, los autores Báez, Blanco y Pérez (2015), Báez, Blanco y Pérez $\left(2015^{\text {b }}\right.$ ), Báez, Pérez y Blanco (2018), Báez, Heredia y Pérez (2017) y Báez Ureña (2018) definieron la formación del concepto en Matemática, apropiación del concepto y formación conceptual

Para explicar la formación del concepto en Matemática, Pérez y Blanco (2019) lo explican desde la lógica de los fundamentos de la investigación, como el proceso activo que transita de lo abstracto a lo concreto, de lo empírico a lo teórico, en el tránsito de lo Inter psíquico (plano social) a lo intrapsíquico (plano individual) lo que constituye su proceso de interiorización.

Este proceso tiene carácter histórico social, y en él se reconoce que, aunque la verdad en Matemática es relativa respecto a determinadas hipótesis, en un campo de esa ciencia ella absoluta en su carácter social. Cuando el concepto es internalizado por el alumno, de forma que lo nuevo sea incorporado a la red conceptual que ya posee, se puede decir que hay apropiación del concepto.

Es así que Báez Ureña (2018) define que la formación conceptual es un proceso en el cual se integran los conceptos apropiados, en una red que se fundamenta en sus propias interrelaciones, a través de la representación y transferencia de sus registros semióticos, la valoración de las hipótesis, el análisis de los movimientos de la variable, el reconocimiento de patrones variacionales del contexto ingenieril, la argumentación de los procesos de variación y cambio, la representación de las relaciones a través de una red identitaria y la consolidación del nexo símbolo-objeto en la resolución de problemas matemáticos aplicados al contexto ingenieril.

Por su parte, los autores Báez, Martínez-López, Pérez y Pérez (2017), Báez, Pérez y Triana (2017) y Báez (2018) definieron al desarrollo conceptual procedimental como el proceso en el que se ponderan las relaciones dialécticas entre el análisis inductivo-deductivo, abstracto- 
concreto, unidad-síntesis, con una organización estructural interna que propicia la construcción lógica de significados en la comprensión de los procesos variacionales, caracterizado por la flexibilidad, significación, gestión y autonomía de los saberes matemáticos, a través de la funcionalidad, contextualización y registros de representación semiótica de lo variacional, de forma tal que mejore el desempeño de los estudiantes en la solución de problemas matemáticos.

Martín, Pérez, Casa y Sánchez (2017), Martín, Pérez y Martínez (2017) y Martín (2018) definieron las relaciones conceptuales como los vínculos que se establecen entre los conceptos, para establecer sistemas que reflejen la estructuración y formalización de la organización del conocimiento, develando el entrelazamiento de los aspectos identitarios y diferenciadores, con significatividad, flexibilidad y autonomía en la representación de la red que se conforma.

Así mismo, Martín (2018) definió el desarrollo de esas relaciones conceptuales como un proceso que se manifiesta en la secuenciación didáctica de logos y praxis orientado a la formalización flexible y significativa de la organización de los vínculos que se establecen entre los conceptos en cualesquiera de sus registros de representación semiótica.

\section{Modelos Didácticos del Cálculo Diferencial y el Álgebra Lineal en el contexto ingenieril}

La modelación teórica constituyó la esencia de las investigaciones doctorales de Báez (2018), Báez Ureña (2018) y Martín (2018) para estudiar las propiedades cualitativas que caracterizan al Cálculo Diferencial y el Álgebra Lineal en el contexto ingenieril, desde sus subsistemas, estructura interna, funciones y relaciones funcionales con un núcleo teórico común: la formación y desarrollo de conceptos.

En el caso del Cálculo Diferencial se trabajó desde dos perspectivas, una modelada por los autores Báez, Blanco y Pérez (2015), Báez, Blanco y Pérez (2015º), Báez, Pérez y Blanco (2018), Báez, Heredia y Pérez (2017) y Báez Ureña (2018), orientada a la formaciónapropiación conceptual para el logro de la cualidad de autonomía conceptual en la resolución de problemas matemáticos de aplicación en el contexto ingenieril (Figura 1), a través de las interrelaciones dialéctico-didácticas que se manifiestan entre los siguientes subsistemas:

- Orientación para la adquisición de recursos en la transferencia de registros de representación semiótica de los conceptos matemáticos.

- Tratamiento conceptual-valorativo de las hipótesis. 
- Apropiación conceptual del movimiento de la variable en la solución de problemas matemáticos de aplicación al contexto ingenieril.

Figura 1 Modelo didáctico de formación-apropiación conceptual en el Cálculo

Diferencial en ingenierías

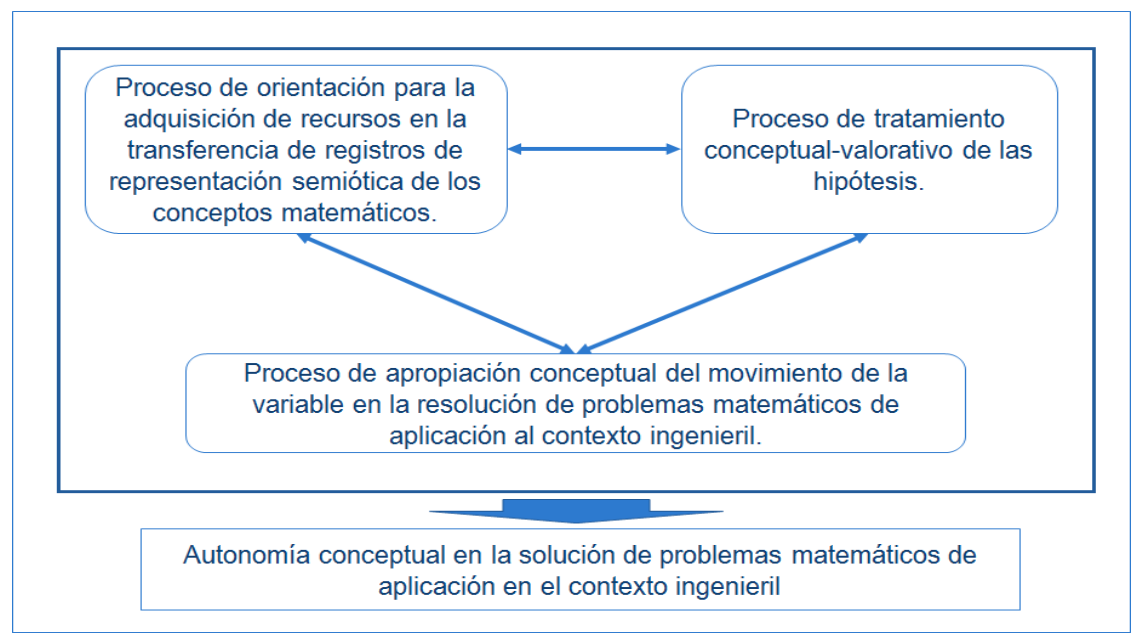

Fuente: Báez Ureña (2018)

La función del subsistema de orientación para la adquisición de recursos en la transferencia de registros semióticos está orientada a la comprensión de la importancia y utilidad de los cambios de representaciones semióticas en la formación y aplicación conceptual, lo que requiere de la flexibilidad en el uso de las transferencias de registros a través de las relaciones de coordinación entre los siguientes componentes.

Este subsistema fue elaborado por Báez, Pérez y Blanco (2018) y Báez Ureña (2018) e incluye los siguientes componentes:

- Discusión reflexiva de los recursos disponibles para la transferencia dentro de un mismo registro de representación.

- Tratamiento de los recursos disponibles para la transferencia en diferentes registros de representación.

- Integración del lenguaje matemático a la comunicación y conversión registro de representación.

Por su parte, la función del subsistema tratamiento conceptual-valorativo de las hipótesis está orientada a la comprensión de la necesidad de valorar las condiciones de existencia de los objetos matemáticos con los que se trabaja, lo que requiere del desarrollo de tres componentes que tengan adecuadas relaciones dialécticas de coordinación y que se caracterice por la cualidad de significatividad de la relación verdad-hipótesis. 
Este subsistema elaborado por Báez Ureña (2018) tiene los siguientes componentes:

- Valoración reflexiva de la relación premisas-ley-consecuencia.

- Reflexividad discursiva en la descripción de los conceptos matemáticos.

- Autovaloración de las condiciones de existencia de los objetos matemáticos.

De forma similar, el subsistema presentado por Báez, Heredia y Pérez (2017) y Báez Ureña (2018), sobre la apropiación conceptual del movimiento de la variable en la solución de problemas matemáticos de aplicación al contexto ingenieril, tiene la función de develar la importancia del movimiento de la variable, manifiesto a través del límite funcional, para la formación, aplicación conceptual y solución de problemas matemáticos del Cálculo Diferencial.

Para ese subsistema, al igual que los anteriores, exige del desarrollo de tres componentes que establecen relaciones dialécticas de coordinación y que se caracterizan por la cualidad de flexibilidad conceptual en el análisis del movimiento de la variable, dichos componentes son:

- Argumentación conceptual del movimiento de la variable en aproximación a una recta.

- Análisis conceptual del movimiento de la variable al infinito.

- Identificación conceptual del movimiento de la variable en aproximación a un punto.

La otra perspectiva de modelación didáctica del Cálculo Diferencial para las carreras de ingeniería, desarrollada por Báez, Martínez-López, Pérez y Pérez (2017) y Báez, Pérez y Triana (2017) se orientó al desarrollo conceptual procedimental para el logro de la cualidad de significatividad lógica del nexo conceptual procedimental (Figura 2), a través de las interrelaciones dialécticas-didácticas que se manifiestan entre los siguientes subsistemas propuestos por Báez (2018) en su investigación doctoral, ellos son:

- Reconocimiento y representación matemática de patrones variacionales del contexto ingenieril.

- Orientación procedimental en la conversión de registros de representación semiótica de los procesos de variación y cambio.

- Orientación conceptual de los procesos de variación y cambio en los contextos geométricos, numéricos, estocásticos y métricos. 
- Articulación de los saberes matemáticos en prácticas sociales en contextos ingenieriles.

Figura 2 Modelo didáctico de desarrollo conceptual procedimental en el Cálculo Diferencial en ingenierías

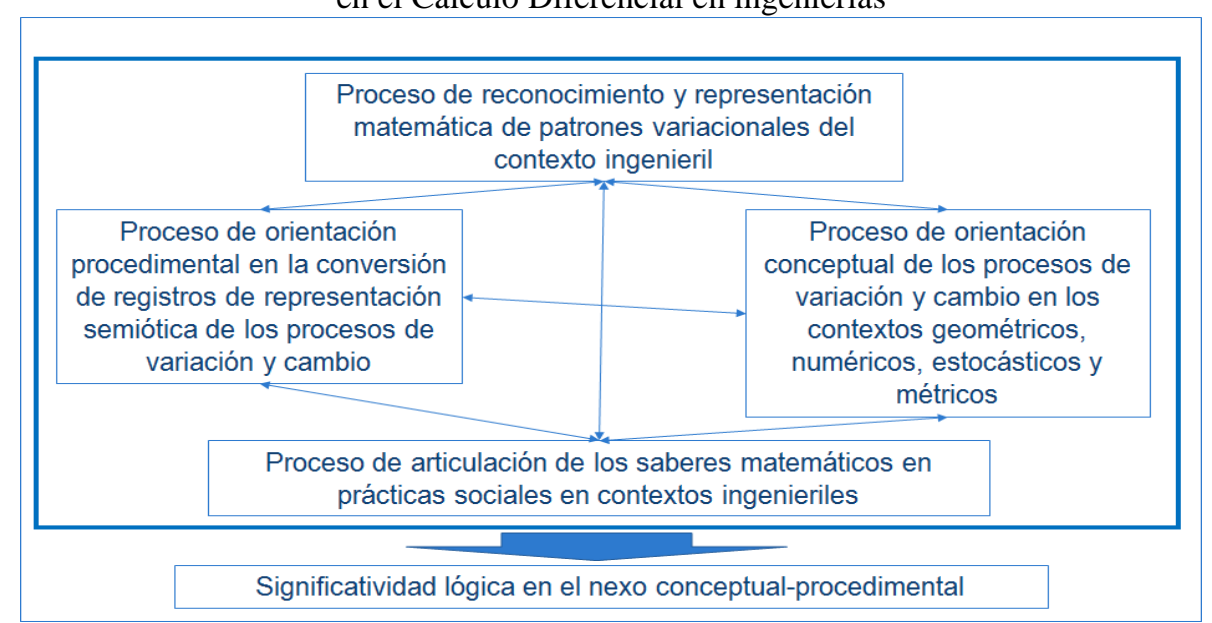

Fuente: Báez Ureña (2018)

La función del subsistema de reconocimiento y representación matemática de patrones variacionales del contexto ingenieril está a la comprensión de las prácticas sociales para de ellas obtener la información necesaria para identificar, clasificar y transformar sus rasgos esenciales en los diferentes registros de representación para favorecer la modelación matemática de dicha situación.

Ese subsistema, presentado por Báez (2018) se caracteriza por la flexibilidad en el reconocimiento de patrones variacionales del contexto ingenieril, como cualidad esencial para el análisis reflexivo en el proceso de solución de los problemas matemáticos, y requiere del desarrollo de relaciones de coordinación entre los siguientes componentes:

- Orientación motivacional reflexiva en la adquisición de datos para el reconocimiento de los fenómenos de patrones en procesos físicos y/o abstracto.

- Identificación de características para la clasificación en patrones de variación y cambio de procesos físicos y/o abstractos.

- Transformación metacognitiva del patrón objeto de estudio en uno de los registros de representación matemática de los procesos de variación y cambio.

El subsistema orientación procedimental en la conversión de registros de representación semiótica de los procesos de variación y cambio, elaborado por Báez, Pérez y Triana (2017), tiene la función de propiciar el razonamiento inductivo-deductivo para la comprensión 
procedimental de los procesos de variación y cambio, y propicia el desarrollo de la flexibilidad inductiva-deductiva en la argumentación de los procesos de variación y cambio como su cualidad resultante, para lo que se requiere desarrollar los siguientes componentes y sus relaciones de coordinación, ellos son:

- Orientación inductiva en las acciones de conversión de los registros de representación de los procesos de variación y cambio.

- Orientación deductiva en la justificación de los procesos de variación y cambio a partir de sus representaciones matemáticas.

- Coordinación reflexiva de los procesos de tratamiento y conversión de los diferentes registros de representación semiótica de los procesos de variación y cambio analizando cómo se manifiesta la dualidad proceso-objeto.

La función del subsistema orientación conceptual de los procesos de variación y cambio está orientada a estimular la caracterización de los rasgos esenciales de los procedimientos y conceptos que intervienen en la situación objeto de estudios a partir de los procesos de variación y cambio en los contextos geométricos, numéricos, estocásticos y métricos y su cualidad resultante es la significatividad de la funcionalidad de los procesos de variación.

Este subsistema, presentado por Báez (2018), se materializa a través de las relaciones de coordinación de los siguientes componentes:

- Orientación ontológica en la funcionalidad de la variación y el cambio en los contextos geométricos, numéricos, estocásticos y métricos.

- Representación de la variación y el cambio como expresión de su funcionalidad.

- Significación de los procesos de variación desde su funcionalidad y su relación con otros conceptos matemáticos.

Y por último, el subsistema articulación de los saberes matemáticos en prácticas sociales en contextos ingenieriles, de la misma autora, tiene la función de generar la interconexión de procedimientos, conceptos y/o significados de prácticas que reflejen situaciones de variación en contextos ingenieriles y la cualidad resultante de resignificación de los saberes matemáticos, para dar progresivamente nuevos significados a los procedimientos y conceptos desde una práctica contextualizada. 
Este subsistema se concreta a través de los siguientes componentes que se coordinan entre sí:

- Reconstrucción reflexiva de significados variacionales desde lo individual y lo social (institucional).

- Explicitación de los procedimientos, conceptos y/o significados que intervienen en las prácticas ingenieriles.

- Movilización reflexiva de los saberes matemáticos para la toma de decisiones con relación a la solución del problema matemático objeto de estudio.

La modelación didáctica del Álgebra Lineal se realizó en dos etapas, en la primera se abordó la comprensión conceptual como vía para la formación y desarrollo de conceptos matemáticos (Mola, 2013), resultado que tuvo su continuidad en una segunda etapa desde la mirada del desarrollo de las relaciones conceptuales con la participación de Martín, Pérez, Blanco y Casas (2014), Martín, Pérez; Casas, Espíndola y Vargas (2015), Martín, Pérez, Casa y Sánchez (2017), Martín, Pérez y Martínez (2017) y Martín (2018).

Es así que el desarrollo de las relaciones conceptuales se modeló con orientación a la significatividad de las relaciones conceptuales en el Álgebra Lineal como cualidad resultante de dicho modelo, y las interrelaciones dialécticas-didácticas que se manifiestan entre los siguientes subsistemas (Figura 3), ellos son:

- Orientación analítica, geométrica y estructural del concepto espacio vectorial.

- Elicitación de las relaciones conceptuales en la secuenciación didáctica entre logos y praxis de la combinación lineal.

- Formalización de la organización conceptual. 
Figura 3 Modelo didáctico de desarrollo de las relaciones conceptuales en el Álgebra Lineal en ingenierías

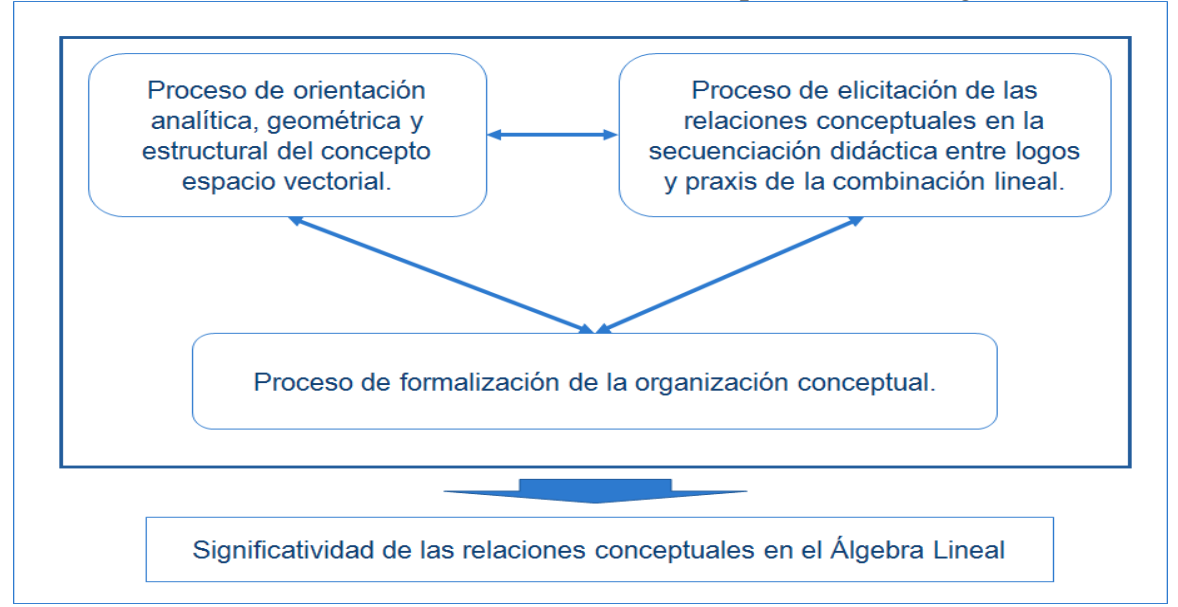

Fuente: Martín (2018)

El subsistema orientación analítica, geométrica y estructural del concepto espacio vectorial elaborado por Martín, Pérez y Martínez (2017), tiene la función de describir el concepto de Espacio Vectorial en todos sus registros de representación semiótica, y en que los vectores de un Espacio Vectorial existen a través de la combinación lineal de vectores, para lograr la flexibilidad en la representación de las relaciones del objeto algebraico como cualidad resultante a través de las relaciones de coordinación de los siguientes componentes:

- Orientación analítica del concepto espacio vectorial.

- Orientación geométrica del concepto espacio vectorial.

- Orientación estructural del concepto espacio vectorial.

- Comprensión de la estructura funcional del concepto espacio vectorial.

Por su parte, el subsistema elicitación de las relaciones conceptuales en la secuenciación didáctica entre logos y praxis de la combinación lineal propuesto por Martín, Pérez, Casa y Sánchez (2017), tiene la función de coordinar las relaciones conceptuales y de representación de lo identitario de cada concepto objeto de estudio del Álgebra Lineal mediante una operación relacionante, que en este caso es la combinación lineal de vectores.

Con este subsistema se debe lograr la flexibilidad en la representación de las relaciones del objeto algebraico a través de la red identitaria, lo que cualifica el proceso de representación de lo identitario de cada concepto objeto de estudio mediante una operación relacionante desde las relaciones de coordinación de los siguientes componentes:

- Representación de las relaciones conceptuales en la combinación lineal. 
- Identificación de los conceptos del álgebra lineal a través de las relaciones con la combinación lineal.

- Representación de la red identitaria de la red de relaciones conceptuales.

- Representación de la red identitaria generadora del sistema conceptual.

Y por último, está el subsistema formalización de la organización conceptual propuesto por Sánchez (2018), con la función de organizar y sistematizar las relaciones conceptuales de manera que incida en la articulación e integración de la formación y desarrollo de conceptos a través de una red conceptual, para lograr la significatividad de la red identitaria como generadora de los conceptos del Álgebra Lineal, que sea resultado de las relaciones de coordinación de los siguientes componentes:

- Comprensión de los conceptos en base a la combinación lineal.

- Reflexividad dialógica sobre las relaciones que definen cada tipo de concepto del Álgebra Lineal.

- Sistematización de los conceptos en base a la combinación lineal.

\section{Pertinencia científica de los modelos teóricos propuestos y sus impactos sociales}

Para determinar el posible conjunto de especialistas considerados expertos en el campo de la didáctica del Cálculo Diferencial y el Álgebra Lineal, se seleccionó de manera directa una población inicial de 43 especialistas en Matemática Educativa, procedentes de Cuba (15), México (11), EEUU (2), Chile (3), Colombia (3) y República Dominicana (9), de los cuales 6 son Maestros en Ciencias y el resto son Doctores en Ciencias.

Se identificó que sólo 35 de ellos tenían un coeficiente de competencia alto, en relación al tema objeto de investigación, y considerando la disposición a participar finalmente quedaron 29 doctores especialistas en Matemática Educativa con un promedio de 10 años de experiencia laboral. Los resultados se procesaron utilizando el Microsoft Excel para analizar la probabilidad de que cada planteamiento fuera ubicado en una categoría determinada.

De los resultados del análisis estadístico se pudo apreciar que, a partir del intercambio con los expertos en relación a los planteamientos que se le consultaron, el resultado de la investigación fue catalogado de muy adecuado con sugerencias que se fueron perfilando en interacción con los expertos. 
Las tres estrategias didácticas diseñadas por Báez (2018), Báez Ureña (2018) y Martín (2018) implementadas en las carreras de ingeniería de la Universidad Autónoma de Santo Domingo constituyeron el sostén de los experimentos pedagógicos orientados a valorar el desempeño de 363 estudiantes para resolver problemas matemáticos en el Cálculo Diferencial (193 estudiantes) y el Álgebra Lineal (170 estudiantes) en el contexto ingenieril.

Los experimentos se realizaron durante el cuarto cuatrimestre del curso escolar 20162017, en tres momentos cada uno: el primero fue el diagnóstico inicial con una prueba de entrada que sirvió para caracterizar a los estudiantes, el segundo incluyó la implementación de las estrategias didácticas, y en el tercero se aplicó la prueba de salida y su comparación con la inicial.

Los resultados de los experimentos fueron socializados por Báez, Martínez-López, Pérez y Pérez (2017), Martín, Pérez y Martínez (2017) y Báez, Pérez y Blanco (2018), en cuyos informes se hace evidente la efectividad de las estrategias didácticas implementadas respecto a la mejoría del desempeño de los estudiantes en la solución de problemas matemáticos del Cálculo Diferencial y el Álgebra Lineal en base a los siguientes criterios de desempeño:

a) Autonomía conceptual en la resolución de tareas de aplicación.

b) Significatividad lógica del nexo conceptual procedimental en la solución de tareas matemáticas.

c) Significatividad de las relaciones conceptuales en la solución de tareas matemáticas.

Finalmente, luego de un amplio proceso de socialización en Congresos, talleres, publicaciones y conferencias, los resultados del proyecto fueron galardonados con el Premio Nacional a los resultados de la investigación científica del año 2018, en la comisión de Ciencias Sociales de la Academia de Ciencias de Cuba.

\section{Conclusiones}

El estudio teórico conceptual develó que la formación y desarrollo conceptual en el Cálculo Diferencial para las carreras de ingeniería, se caracteriza por la autonomía conceptual en la solución de problemas matemáticos aplicados al contexto ingenieril, y por la significatividad lógica en el nexo conceptual procedimental como vía para mejorar el desempeño de los estudiantes en la solución de problemas matemáticos. 
De modo que, la autonomía conceptual en la resolución de tareas de aplicación se materialice en el uso de las transferencias de registros semióticos de los objetos matemáticos; la significatividad de la relación verdad-hipótesis y la flexibilidad conceptual en el análisis del movimiento de la variable.

Y que, la significatividad lógica del nexo conceptual procedimental se concrete en la flexibilidad en el reconocimiento de patrones variacionales del contexto ingenieril; el análisis inductivo-deductivo de la argumentación de los procesos de variación y cambio; la significatividad de la funcionalidad de dichos procesos y la resignificación de los saberes matemáticos en el contexto ingenieril.

A su vez, para el Álgebra Lineal la formación y desarrollo conceptual se caracteriza por la significatividad de las relaciones conceptuales y se materializada en la flexibilidad en la representación de las relaciones de los conceptos matemáticos, la flexibilidad en la representación de los conceptos matemáticos a través de la red identitaria y la significatividad de la red identitaria como generadora de los conceptos matemáticos.

El éxito práctico del tratamiento didáctico de las relaciones, formación y desarrollo conceptual-procedimental exige de la preparación matemática de los docentes, sus habilidades para utilizar las tecnologías en función de la docencia, sus conocimientos sobre el objeto de estudio de los ingenieros, su comprensión sobre la función didáctica del tratamiento y conversión de los registros de representación semiótica y sobre la necesidad de formar en los estudiantes el uso correcto del lenguaje matemático, como elemento fundamental de interacción social en la actividad de estudio.

Una adecuada preparación de los ingenieros en formación en el Cálculo Diferencial y el Álgebra Lineal, como primeras asignaturas de Matemática en su formación ingenieril, sienta las bases para la sólida formación básica que requieren los ingenieros del futuro.

La construcción teórica ha sido un proceso de indagación continua en el que se reconoce la importancia de la objetividad del conocimiento constatado en la práctica, conscientes de que las conclusiones obtenidas en la investigación están sujetas a comprobaciones, enriquecimiento y perfeccionamiento a partir de su aplicación en diversos contextos históricos y culturales. 


\section{Agradecimientos}

Agradezco el apoyo y participación de los investigadores del Proyecto Perfeccionamiento de la enseñanza de la Matemática del Programa Nacional Problemas Actuales del Sistema Educativo Cubano, perspectivas de desarrollo (código PP221LH053), en especial al Dr. C. Ramón Blanco Sánchez y la Dr. C. Nancy Montes de Oca Recio, sin su participación este proyecto no hubiera sido posible, y a los Doctores Neel Lobatchewski Báez Ureña, Ana Mercedes Báez y Ángela Mercedes Báez Sánchez, docentes de la Universidad Autónoma de Santo Domingo y participantes en el proyecto como estudiantes de doctorado.

\section{Referencias}

Arias, G. (2018). La integralidad del método dialéctico en la Teoría Histórico Cultural. Psicología Escolar e Educacional, 22(3), pp. 631-641. https://doi.org/10.1590/21753539201803002 .

Arrieta, J. y Díaz, L. (2015). Una perspectiva de la modelación desde la Socioepistemología. Revista latinoamericana de investigación en matemática educativa, 18(1), pp. 19-48. http://relime.org/index.php/numeros/todos-numeros/volumen-18/numero-18-1/399$201501 \mathrm{a}$

Báez Ureña, N. (2018, julio 12). Estrategia didáctica para la formación de conceptos en el proceso enseñanza-aprendizaje del Cálculo Diferencial de una variable real en las carreras de ingeniería [Tesis de doctorado]. Universidad de Camagüey "Ignacio Agramonte Loynaz". Camagüey.

Báez, A. (2018, junio, 26). Estrategia didáctica para el desarrollo conceptual procedimental en el Cálculo Diferencial de una variable real, para las carreras de ingeniería [Tesis de doctorado]. Universidad de Camagüey "Ignacio Agramonte Loynaz". Camagüey.

Báez, A., Pérez, O; Triana, B. (2017). Propuesta didáctica basada en múltiples formas de representación semiótica de los objetos matemáticos para desarrollar el proceso de enseñanza aprendizaje del cálculo diferencial. Academia y Virtualidad, 10(2), pp. 20-30. https://dx.doi.org/10.18359/ravi.2743

Báez, A; Martínez-López, Y; Pérez, O; Pérez, R. (2017), Propuesta de tareas para el desarrollo del pensamiento variacional en estudiantes de ingeniería. Formación Universitaria. 10(3), pp. 93-106. http://dx.doi.org/10.4067/S0718-50062017000300010

Báez, N., Pérez, O; Blanco, R. (2018). Los registros de representación semiótica como vía de materialización de los postulados vigotskianos sobre pensamiento y lenguaje. Academia y Virtualidad, 11(1). pp. 16-26. https://doi.org/10.18359/ravi.2885

Báez, N; Blanco, R; Pérez, O: (2015). Fundamentación teórica de la apropiación conceptual con ayuda de las TIC ejemplificado en la derivada. Acta Latinoamericana de Matemática Educativa, 28, pp. 1576-1582. http://www.clame.org.mx/documentos/alme28.pdf

Báez, N; Blanco, R; Pérez, O: $\left(2015^{\text {b }}\right)$. Dificultades de los alumnos en el trabajo con los conceptos del Cálculo Diferencial. Acta Latinoamericana de Matemática Educativa, 28, pp. 57-63. http://www.clame.org.mx/documentos/alme28.pdf 
Báez, N; Heredia, W; Pérez, O. (2017). El movimiento de la variable en el cálculo diferencial: orientaciones didácticas. Transformación, 13(3), pp. 444-455. http://scielo.sld.cu/scielo.php?script=sci_arttext\&pid=S2077-29552017000300011

Bertalanfly, L. [Ed.]. (1954). Teoría general de sistemas, Nueva York: Editorial Fondo de Cultura Económica.

Bueno, S; Pérez, O. (2018). La idoneidad epistémica del concepto función real de una variable real en carreras de ingenierías. Revista Educación Matemática. 30(2), pp. 202-231. http://dx.doi.org/10.24844/EM3002.8

Camacho-Ríos, A. (2011). Socioepistemología y prácticas sociales. Hacia una enseñanza dinámica del cálculo diferencial. Revista Iberoamericana de Educación Superior, 2(3), pp. 152-171. https://doi.org/10.22201/iisue.20072872e.2011.3.32

Cantoral, R. [Ed.] (2013). Teoría socioepistemológica de la matemática educativa. Estudios sobre construcción social del conocimiento (1st ed.), Barcelona: Editorial Gedisa SA

Castellanos, D., Castellanos, B., Llivina, M., Silverio, M., Reinoso, C., y García, C. [Ed.] (2002). Aprender y enseñar en la escuela. Una concepción desarrolladora, Habana: Editorial Pueblo y Educación.

Cordero, F., Del Valle, T. y Morales, A. (2019). Usos de la optimización de ingenieros en formación: El rol de la ingeniería mecatrónica y de la obra de Lagrange. Revista Latinoamericana de Investigación en Matemática Educativa, 22 (2), pp. 185 - 212. https://doi.org/10.12802/relime.19.2223

D'Amore, B. (2009). Conceptualización, registros de representaciones semióticas y noética: interacciones constructivistas en el aprendizaje de los conceptos matemáticos e hipótesis sobre algunos factores que inhiben la devolución. Revista científica, 11, pp. 150-164. https://dialnet.unirioja.es/servlet/articulo?codigo $=820883$

Farit, J; Estrada, P; Ramos, I (2017). Una concepción y modo de gestión didáctica de la matemática en la carrera de ingeniería civil. Transformación, 13(1), pp. 114-129. http://scielo.sld.cu/scielo.php?script=sci_abstract\&pid=S2077-29552017000100012

Godino, J., Batanero, C. y Font, V. (2007). The ontosemiotic approach to research in mathematics education. ZDM. The International Journal on Mathematics Education, 39(12), pp. 127-135. https://link.springer.com/article/10.1007/s11858-006-0004-1

Godino, J., Vicenç, F., Contreras, A. y Wilhelmi, M. (2005). Una visión de la didáctica francesa desde el enfoque ontosemiótico de la cognición e instrucción matemática. Revista Latinoamericana de Matemática Educativa, 9(1), pp. 117-150. http://www.scielo.org. $m x /$ scielo.php? script $=$ sci_arttext\&pid $=$ S1665-24362006000100006

González, V. (1984). El enfoque sistémico en los medios de enseñanza. Revista Cubana de Educación Superior. 4(1), pp. 10-21.

King, C. (2012). Restructuring Engineering Education: ¿Why, How and When?. Journal of Engineering Education. $\quad 101(1), \quad$ pp. https://onlinelibrary.wiley.com/doi/abs/10.1002/j.2168-9830.2012.tb00038.x

León-Duarte, G. (2019). Aportes teóricos a la investigación del campo periodístico. Sentidos y significados desde el Campo Intelectual Creador. Intercom: Revista Brasileira de Ciências 
da Comunicação, São Paulo, 42(3), pp. 41-59. http://www.scielo.br/scielo.php?pid=S1809$\underline{58442019000300041 \& \text { script }=\text { sci_arttext }}$

Marrero, O. y Lasso, M. (2017). El proceso de enseñanza-aprendizaje por competencias. Una visión desde el enfoque sistémico. Revista Congreso Universidad. 6(4), pp. 28-46. http://revista.congresouniversidad.cu/index.php/rcu/article/view/837

Martín, A. (2018, junio, 26). Estrategia didáctica para el desarrollo de relaciones conceptuales en el Álgebra Lineal para las carreras de ingeniería [Tesis de doctorado]. Universidad de Camagüey "Ignacio Agramonte Loynaz". Camagüey.

Martín, A. y Pérez, O. (2019). Las ciencias básicas en la formación del ingeniero: el caso del Álgebra Lineal. Revista Estudios Generales UNAPEC. 2(4), pp. 31-38. https://www.researchgate.net/publication/339663530_LAS_CIENCIAS_BASICAS_EN_L A_FORMACION_DEL_INGENIERO_EL_CASO_DEL_ALGEBRA_LINEAL

Martín, A., Pérez, O., Blanco, R., Casas, L. (2014). Los espacios vectoriales, como estructuras algebraicas, en el proceso de enseñanza aprendizaje del Álgebra Lineal: una propuesta de investigación. Acta Latinoamericana de Matemática Educativa. 27, pp. 1073-1082. http://www.clame.org.mx/documentos/alme27.pdf

Martín, A; Pérez, O. y Martínez, Y. (2017). Propuesta didáctica para la enseñanza del concepto espacio vectorial. REFCalE: Revista Electrónica Formación y Calidad Educativa, 5(2), 195-209. http://refcale.uleam.edu.ec/

Martín, A; Pérez, O; Casa, L; Sánchez, R, (2017). Secuenciación didáctica entre logos y praxis de la combinación lineal. Ciencias Matemáticas. 31(2), pp. 143-149. https://www.matcom.uh.cu

Martín, A; Pérez, O; Casas, L; Espíndola, A; Vargas, A. (2015). ¿Contribuye la didáctica del Álgebra Lineal a que los estudiantes identifiquen los espacios vectoriales como una estructura sistémica? Acta Latinoamericana de Matemática Educativa. 28, pp. 315-322. http://www.clame.org.mx/documentos/alme28.pdf

Mendoza, F. y Escalona, M. (2019). Contextualización de la enseñanza de la Matemática en la carrera de Ingeniería Civil. ROCA. Revista científico-educacional de la provincia Granma. 15(3), pp. 13-24. https://revistas.udg.co.cu/index.php/roca/article/view/924

Minnaard, C. y Comoglio, M. (2019). Aplicaciones de la simulación en la enseñanza de la ingeniería. Congreso Iberomaricano de Educación Científica. Uruguay. https://digital.cic.gba.gob.ar/handle/11746/10061

Mola, C. (2013, enero 23). Estrategia didáctica para la comprensión de los objetos del álgebra lineal en las carreras de ingeniería de la universidad de Camagüey [Tesis de doctorado]. Universidad de Camagüey "Ignacio Agramonte Loynaz". Camagüey.

Nardín, A; Montalván, M; Salgado, M; Pérez, O. (2017). Errores de los estudiantes en el tema de derivada de funciones de varias variables. Revista Paradigma. 38(1), pp. 312 - 329. http://revistas.upel.edu.ve/index.php/paradigma/article/view/5715/3041

Pérez, O. (2018). La Matemática Educativa en Camagüey: incidencia social de un programa de maestría. Revista Latinoamericana de Investigación en Matemática Educativa, 21 (2), pp. 125-130. https://dx.doi.org/10.12802/relime.18.2120 
Pérez, O. y Blanco, R., (2019). Contribución teórica y práctica a la didáctica del Cálculo Diferencial y del Álgebra Lineal para carreras de ingeniería. Anales de la Academia de $\begin{array}{lllll}\text { Ciencias de } & \text { Cuba. } & \text { 9(3). } & \text { pp. }\end{array}$ http://www.revistaccuba.cu/index.php/revacc/article/view/692

Plaza, L. (2016). Obstáculos presentes en Modelación Matemática. Caso Ecuaciones Diferenciales en la formación de Ingenieros. Revista Científica. 2(25), pp. 176-187. Recuperado http://revistas.udistrital.edu.co/ojs/index.php/revcie/article/view/10340/11774

Plaza, L. y Villa-Ochoa, J. (2019). Obstáculos matemáticos detectados en la formación de ingenieros. Una revisión de literatura. Revista Virtual Universidad Católica del Norte. (58), pp. 223-241. DOI: https://doi.org/10.35575/rvucn.n58a13

Ramos, G. (2000). Filosofía y actividad: implicaciones para la formación humanística del profesional universitario de las Carreras Técnicas [Tesis de doctorado]. Universidad de La Habana, Habana.

Rendón-Mesa, P., Duarte, P. y Villa-Ochoa, J. A. (2016). Articulación entre la matemática y el campo de acción de la ingeniería de diseño de producto: componentes de un proceso de modelación matemática. Revista de La Facultad de Ingeniería U.C.V., 31(2), pp. 21-36. Recuperado de http://funes.uniandes.edu.co/11854/

Reyes-Gasperini, D. y Cantoral, R. (2014). Socioepistemología y empoderamiento: la profesionalización docente desde la problematización del saber matemático. Boletim de Educação Matemática. 28(48), pp. 360-382. http://dx.doi.org/10.1590/1980$4415 \mathrm{v} 28 \mathrm{n} 48 \mathrm{a} 14$

Rodríguez, R. (2017). Repensando la enseñanza de las matemáticas para futuros ingenieros: actualidades y desafíos. Revista de Investigación Educativa de la REDIECH. 8(15), pp. 6985. https://www.rediech.org/ojs/2017/index.php/ie_rie_rediech/article/view/55

Rosental, M., y Ludín, P. [Ed.]. (1981). Diccionario Filosófico, Habana, Cuba: Editorial Revolucionaria.

Tall, D. [Ed.] (1991). Advanced Mathematical Thinking, Dordrecht: Kluwer A. P.

Valledor, R. (2019). La innovación en la investigación educacional. La innovación Teórica. Didasc@lia: Didáctica $\quad y \quad$ Educación. $\quad 10(4), \quad$ pp. $\quad$ 17-32. http://revistas.ult.edu.cu/index.php/didascalia/article/view/1354

Vargas, A Burguet, I; Lezcano, L; Durán, M. (2018). Las relaciones intradisciplinarias en el currículo de la carrera ingeniería en ciencias informáticas: una visión desde el Álgebra Lineal. Acta Latinoamericana de Matemática Educativa. 31, pp. 1209-1216. http://www.clame.org.mx/documentos/alme31.pdf

Vigotsky, L. [Ed.]. (1987). Historia del desarrollo de las funciones psíquicas superiores, Habana: Editorial Científico Técnica. 